\title{
Hepatitis E: Canada welcomes the world
}

C ASE REPORTS PRESENTED IN THIS ISSUE OF THE JOURNAL represent the first documented cases of hepatitis $E$ in Canada (pages 34-37 and 39-41). Globally, the existence of enterically transmitted non-A, non-B hepatitis has been recognized for many years and accounts for over $50 \%$ of acute hepatitis in developing nations $(1,2)$. Usual hepatotropic viruses were not found to cause this disease and the term viral hepatitis type $E$ (HEV) was coined. Although studies in nonhuman primates failed to yield a culturable pathogen, electron microscopy of stool samples revealed the presence of viruslike particles (1). Other studies suggested that HEV was due to an RNA virus, and isolation of viral complementary DNA clones led to demonstration of viral RNA genomes in infected subjects $(3,4)$. Subsequent analysis demonstrated that HEV is a 27 to $32 \mathrm{~nm}$ nonenveloped virus with a single-strand positive sense polyadenylated RNA genome representative of a new family of hepatotropic viruses. With the use of recombinant proteins from expression vectors, diagnostic tests capable of identifying hepatitis $E$ infection were developed and confirmed that a single agent, HEV (still uncultured), was responsible for the majority of non-A, non-B acute hepatitis outbreaks in developing countries. Molecular biology techniques could be used to detect viral genomes in liver tissue, serum and stool during the illness. These techniques should allow better characterization of the natural history of HEV infection (1).

The clinical and epidemiological features of HEV have recently been reviewed $(1,2)$. Estimates suggest that up to 2 million cases occur yearly in the Indian subcontinent. HEV causes large epidemics of acute self-limited icteric hepatitis often linked to ingestion of contaminated water. More recent data suggest that HEV is also a common cause of sporadic hepatitis in developing countries, especially in children. Seroprevalence for HEV antibodies can approach $25 \%$ in children from endemic areas despite less frequent icteric hepatitis (5). Person to person transmission is less well characterized but may be responsible for some cases of HEV (2). Icteric HEV presents clinically as a typical acute viral hepatitis with prodromal and icteric phases. Patients with icteric HEV tend to be older (15 to 40 years of age versus less than 10 years of age), have longer incubation periods (mean 40 days versus 30 days) and higher case fatality rates ( 0.5 to $4 \%$ versus 0.1 to $2.7 \%$ ) than patients with hepatitis A virus from similar geographic areas. HEV is also responsible for many cases of fulminant hepatitis in endemic regions. Pregnant females appear to be particularly susceptible to HEV, especially when acquired in the third trimester. Fulminant hepatitis (over $30 \%$ of HEV infections in some studies) and high case fatality rates (up to $20 \%$ ) occur in this patient population, the basis of which remains unclear. Liver biopsy changes of cholestasis occur with greater frequency than in hepatitis A (at least $50 \%$ of cases) but pathological findings are otherwise typical of acute viral hepatitis (1). Loss of detectable viral genomes in tissue, serum and stool parallels clinical resolution of hepatitis and return of liver histology to normal. Treatment is purely supportive and chronic hepatitis does not occur.

Testing for hepatitis $E$ exposure and active infection has improved substantially in recent years (1,5-7). Polymerase chain reaction (PCR) analysis for HEV genomes in serum, stool and liver tissue has supplanted evaluation of the stool by electron microscopy as the definitive test for active infection $(3,4,8)$. Unfortunately, the availability of this test is limited to research facilities. Serological tests for antibodies to recombinant HEV proteins are available at specialized centres and are being used to clarify the epidemiological and clinical features of HEV $(1,6,7)$. Both the enzyme immunoassay (sensitivity 78 to $100 \%$ ) and immunoblot (sensitivity 85 to $100 \%$ ) tests are sensitive for acute icteric HEV. Immunoglobulin (lg) M titres rise early and can be sustained for over six months. The duration of the $\lg G$ response remains in question. Some studies suggest that IgG reactivity diminishes within a year of infection (9). The specificity of these antibody tests is uncertain. False positive serological tests can occur in other forms of liver disease (8). This makes interpretation of HEV prevalence data from developed countries difficult. No large scale study has compared antibody reactivity with PCR analysis for HEV genomes in cases of suspected HEV.

The inability to culture wild-type or attenuated HEV virus has impeded development of a vaccine. Recent results using recombinant proteins in experimental animals are promising (10). Immunoglobulin prophylaxis has yet to prove effective. Adequate hygiene and avoidance of contaminated water sources are the only preventive measures available.

Although HEV represents a major health problem for developing nations, it is not a major clinical problem in North America $(1,7)$. Cases such as those presented in the Journal serve to emphasize the potential for HEV acquisition by North American travellers. Sporadic cases of hepatitis $E$ have been reported in developed countries, most often in persons returning from endemic areas (11). Recovery is usual and recent studies suggest that HEV is not a major cause of fulminant hepatitis or death from liver disease in the United States (and presumably Canada) (8). Seroprevalence for HEV is low in the 
United States (less than $0.5 \%$ ) and the predictive value of positive tests for HEV infection is uncertain in this population (7). No common source outbreaks have been reported in the United States or Canada. As such, the role of HEV in North American liver disease is likely to be negligible. However, one must note that large studies using sensitive and specific assays for HEV are only now being initiated. The pathogenesis and epidemiology of this virus remain to be fully defined. Preliminary reports from Europe suggest that up to $6 \%$ of acute non-A, non-B hepatitis patients have anti-HEV antibodies and that travel to endemic areas is not always noted $(12,13)$. These observations, coupled with the increased scope and frequency of global travel, may prove the optimistic predictions regarding the impact of HEV in North America incorrect.

In summary, HEV is a novel hepatotropic virus responsible for the majority of enterically spread non-A, non-B hepatitis in developing countries and represents a major health concern in these countries. HEV plays a limited role in clinical liver disease in Canada and the United States and, except for sporadic cases, is unlikely to represent a major health threat in these countries. Travellers to endemic areas should be advised to take sanitary precautions to decrease the risk of acquiring either hepatitis $E$ or hepatitis $A$. Pregnant females travelling to endemic areas should be especially aware of the potential risk. Given the lack of effective immune globulin preparations or vaccine, guaranteeing adequate sanitary conditions is the only prophylactic measure that decreases the risk of acquiring HEV. If this cannot be guaranteed, then high risk patients should delay travel until well into the postpartum period. Developing techniques in molecular biology and viral identification should improve our epidemiological and clinical knowledge base and hopefully will lead to development of an effective HEV vaccine.

\section{REFERENCES}

1. Krawczynski K. Hepatitis E. Hepatology 1993;17:932-41.

2. Purdy M, Krawczynski K. Hepatitis E. Gastroenterol Clin North Am 1994;23:537-46.

3. Reyes G, Purdy M, Kim J, et al. Isolation of a cDNA from the virus responsible for enterically transmitted non-A, non-B hepatitis. Science 1990;247:1335-9.

4. McCaustland K, Bi S, Purdy M, Bradley D. Application of two RNA extraction methods prior to amplification of hepatitis $E$ virus nucleic acid by polymerase chain reaction. J Virol Methods 1991;35:331-42.

5. Paul D, Knigge M, Ritter A, et al. Determination of hepatitis $E$ virus seroprevalence by using recombinant fusion proteins and synthetic peptides. J Infect Dis 1994;169:801-6.

6. Favorov M, Khudyakov $\mathrm{Y}$, Fields $\mathrm{H}$, et al. Serologic identification of hepatitis $E$ virus infections in epidemic and endemic settings. J Med Virol 1992;36:246-50.

7. Favorov M, Khudyakov Y, Fields H, Khudyakova N, Padhye N, Alter M. Enzyme immunoassay for the detection of antibody to hepatitis $\mathrm{E}$ virus based on synthetic peptides. J Virol Methods 1994;46:237-50.

8. Kuwada S, Patel V, Hollinger F, et al. Non-A, non-B fulminant hepatitis is also non-E and non-C. Am J Gastroenterol 1994;89:57-61.

9. Lok A, Kwan W, Moeckli R, et al. Seroepidemiological survey of hepatitis $E$ in Hong Kong by recombinant-based enzyme immunoassays. Lancet 1992;340:1205-8.

10. Purdy M, McCaustland K, Krawczynski K, Spelbring J, Reyes G. Preliminary evidence that a trpE-HEV fusion protein protects cynomolgus macaques against challenge with wild-type hepatitis E virus (HEV). J Med Virol 1993;41:90-4.

11. Centers for Disease Control. Hepatitis $E$ among US travelers. MMWR 1993;42:1-4.

12. Zaaijer H, Kok M, Lelie P, Timmerman R, Chau K, VanderPaul H. Hepatitis $E$ in the Netherlands: imported and endemic. Lancet 1993;341:826.

13. Zanetti A, Dawson G. Hepatitis type $E$ in Italy: a seroepidemiological survey. Study Group of Hepatitis E. J Med Virol 1994;42:318-20.

Barry G Rosser MD FRCPC Liver Diseases Unit, Section of Gastroenterology University of Manitoba Winnipeg, Manitoba 


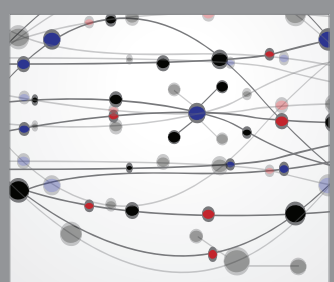

The Scientific World Journal
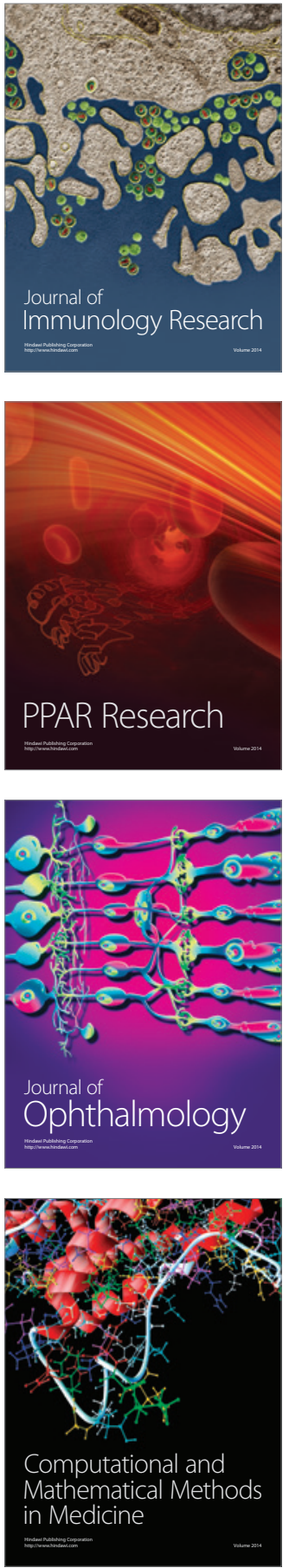

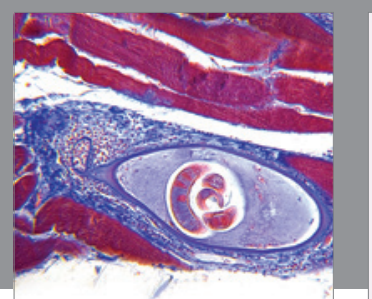

Gastroenterology Research and Practice

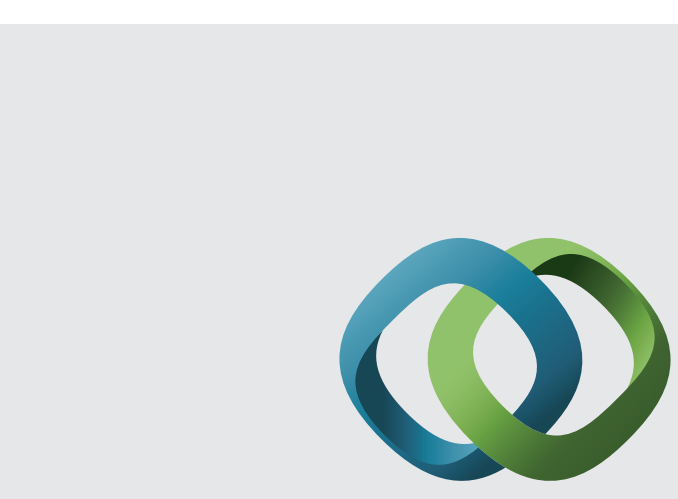

\section{Hindawi}

Submit your manuscripts at

http://www.hindawi.com
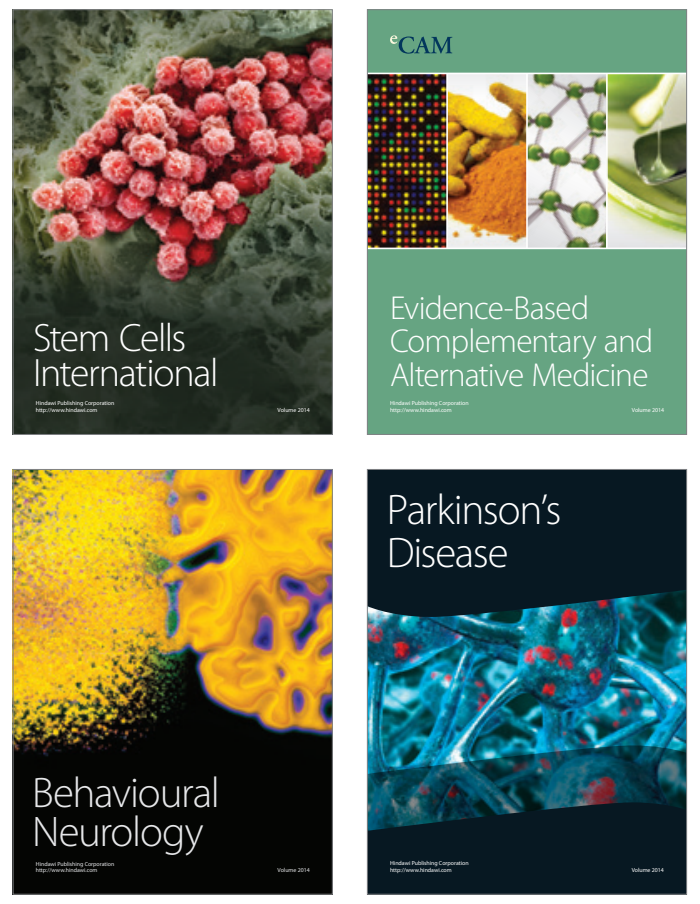
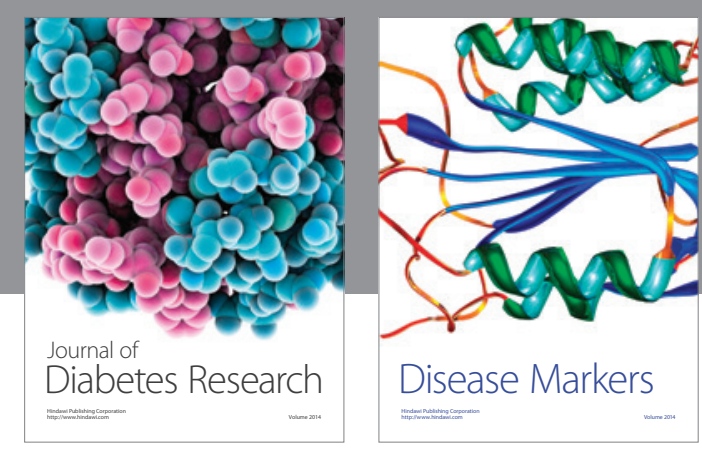

Disease Markers
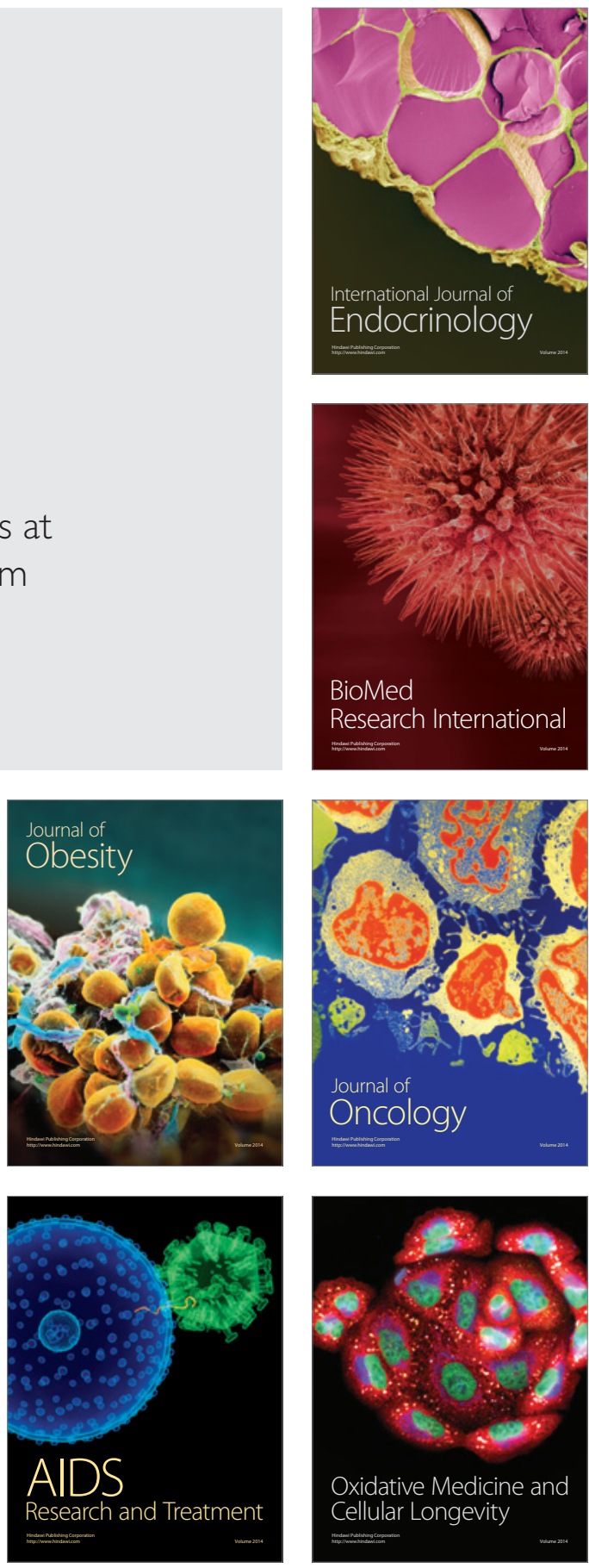